\title{
EFECTIVIDAD DE LAS REDES DE NIEBLA PARA DETERMINAR LA RIQUEZA DE AVES EN UN BOSQUE MONTANO DE LOS ANDES CENTRALES (SALENTO, QUINDÍO, COLOMBIA)
}

\author{
MIST NET EFFECTIVENESS TO DETERMINE BIRD RICHNESS IN A MONTANE \\ FOREST OF CENTRAL ANDES (SALENTO, QUINDÍO, COLOMBIA)
}

Juliana M. Polanco ${ }^{1}$, Albert Ospina Duque ${ }^{1}$, Daniel Arango Giraldo ${ }^{1}$, Jhose Snaider Granada ${ }^{1}$, Oscar Humberto Marín Gómez ${ }^{1}$

\footnotetext{
${ }^{1}$ Programa de Licenciatura en Biología y Educación Ambiental. Grupo de Biodiversidad y Educación Ambiental (BIO EDUQ). Universidad del Quindío

Recibido: 09 de Febrero de 2012

Aceptado: 10 de Marzo de 2015

*Correspondencia del autor: Universidad del Quindío. A.A. 460. Armenia, Quindío. E-mail: ohmaring@unal.edu.co
}

\begin{abstract}
RESUMEN
Las redes de niebla han sido utilizadas como un método importante para realizar inventarios de aves en varios hábitats neotropicales, pero su efectividad con respecto a otros métodos como los puntos de conteo, no ha sido evaluada extensivamente. Este trabajo compara la efectividad de las redes de niebla y del método de puntos de conteo en la determinación de la diversidad de aves en un bosque montano de los Andes centrales de Colombia. Durante 6 meses en un bosque montano del departamento de Quindío, con un esfuerzo de captura de 2630 horas red se registraron 608 individuos de 81 especies. Con el método de puntos de conteo encontraron 106 especies. Sin embargo, 31 especies fueron detectadas solo por capturas en redes. La mayor tasa de captura ocurrió durante las primeras horas de la mañana y al medio día (06:00-09:00 h y de 12:00-13:00h) mientras que en la tarde la tasa de captura disminuyó. Concluimos que por el método de puntos de conteo existe una mayor probabilidad en llevar a cabo un inventario representativo en menos tiempo, por otro lado el papel de las redes en los inventarios se consideraría complementario, siendo su uso necesario para conseguir otros tipos de información, como mediciones morfológicas, indicios de reproducción y muda.
\end{abstract}

Palabras Clave: bosque montano, capturas, efectividad, puntos de conteo, riqueza de aves, 


\begin{abstract}
Mist nets have been used to conduct bird inventories in several Neotropical habitats, but rarely their effectiveness have been evaluated in comparison to count points. This study compares the effectiveness of the mist nets and count points in determining the diversity of montane forest birds in montane forest of the Colombian Central Andes. During 6 months, we used mist nets in different habitats with a capture effort of 2630 net-hours. 608 individuals of 81 species of birds were recorded. In the point counts 106 species of birds were detected. However we captured 31 species that were not recorded in the count points. Higher capture rate occurred during the early hours of the morning and at noon (06:00-09:00 $\mathrm{h}$ and 12:00-13:00 $\mathrm{h}$ ) while in the afternoon the catch rate declined. From our data and the comparison with the data of other authors, we conclude that point counts are useful to obtain a representative bird inventory in less time than using mist nets alone. However, the role of mist nets in inventories must be considered as complementary. And they are necessary to obtain other information such as morphological measurements reproduction clues and molt.
\end{abstract}

Keywords: bird richness, captures, effectiveness, montane forest, point counts.

\section{INTRODUCCIÓN}

Las redes de niebla han sido utilizadas para capturar aves y monitorear sus poblaciones. Aunque el número de individuos capturados en las redes se ha usado para estimar tamaños poblacionales de ciertas especies, esta aproximación puede presentar varios problemas. Por una parte, para estimar abundancias, el uso de puntos de conteo proporciona resultados más precisos, ya que los datos de la captura con redes provienen de pocos puntos por unidad de tiempo (1).

Además, como cualquier método de muestreo por captura pasiva, el número de aves capturadas en las redes de niebla está fuertemente influenciado por la actividad de las aves $(1,2)$. La efectividad de las redes de niebla depende de varios factores como: el tamaño de la malla (3); el tipo y calidad de material con el que está fabricada (4), así como su desgaste; la visibilidad y las condiciones meteorológicas $(4,5,6)$; el tipo de hábitat y la estructura de la vegetación (5); el tamaño de las aves (6); el tipo de vuelo, el comportamiento territorial (1); y la habilidad del investigador para manejar las redes correctamente (7). A pesar de que estos sesgos son ampliamente conocidos, algunos estudios no mencionan su corrección (8), lo que puede causar inconsistencias en los cálculos de valores de abundancia y riqueza de especies $(1,7)$.

En varios trabajos de inventarios y consultorías ambientales se ha utilizado este método para comparar la diversidad y estructura trófica de la avifauna de distintas áreas o coberturas vegetales, pero no se ha tenido en cuenta sus limitaciones (6). Por lo tanto, el uso de los datos obtenidos por medio de capturas con redes de niebla se ha basado solamente en suposiciones de las frecuencias de captura y las abundancias relativas de las especies de aves (7). Esto ha llevado a algunos investigadores a identificar los factores que causan variación en las tasas de captura, ya que éstos tienen consecuencias en las estimaciones del tamaño de la población (8).

Aunque las redes de niebla han sido utilizadas como única herramienta para realizar inventarios de aves en varios bosques neotropicales $(8,9)$, ya que reducen en gran medida el sesgo del observador, en comparación con los métodos puntos de conteo y grabación de vocalizaciones (10); hay varios estudios que han evaluado su efectividad en relación con otros métodos $(7,11)$. Dado que la experiencia del observador es un aspecto critico que influye en la efectividad de cada método $(4,7,12)$ En el presente estudio, un grupo de cuatro investigadores con un entrenamiento básico (seis meses) en métodos de muestreo e identificación de aves, evaluamos la efectividad de las redes de niebla en la determinación de la riqueza de aves con relación al método puntos de conteo en un bosque montano. Para esto, comparamos la riqueza y el número de detecciones de las aves capturadas con redes de niebla vs los registros obtenidos con el método de puntos de conteo (detecciones visuales y auditivas). Además, clasificamos las aves según su tamaño corporal, grupo trófico y gremios de forrajeo para examinar si hay diferencias entre los dos tipos de métodos empleados. 


\section{MATERIALES Y MÉTODOS}

\section{Área de estudio}

Realizamos este estudio en la Finca Los Andes (4 $41^{\prime} 33,4^{\prime \prime} \mathrm{N}$ y $75^{\circ} 33^{\prime} 12,5^{\prime \prime O}$; 2160-2600 m.s.n.m), cuenca media del río Quindío, en la vereda Boquía, municipio de Salento, Quindío. Esta zona incluye la Reserva Natural La Patasola y limita al norte con el Santuario de Flora y Fauna Otún Quimbaya, Risaralda (13). El paisaje se caracteriza por la presencia de fragmentos de bosque muy húmedo montano (bmh$\mathrm{MB})$ inmersos en una matriz intervenida de potreros y plantaciones de pino (Pinus sp.) y eucalipto (Eucalyptus sp) que abarcan un área 703 ha. La precipitación media anual oscila entre 2.200 y $2.400 \mathrm{~mm}$, las épocas más lluviosas se presentan entre marzo- mayo, y entre septiembre y diciembre. La época seca con lluvias ocasionales se extiende de enero a marzo y de julio a agosto. La temperatura media anual es de $18^{\circ} \mathrm{C}$ y la humedad relativa es del 75\% (13).

El bosque presentaba un dosel de 15 a $20 \mathrm{~m}$ de alto, con dominancia de Ocotea sp., Nectandra sp., Cecropia telealba, Otoba lehmanii, Wettinia kalbreyeri, Aegiphila sp., Guettarda sp., Brosimum utile, Weinmannia tomentosa, Quercus humboldtii, Juglans neotropica y Ceroxylon quinduense. El sotobosque era relativamente denso con un alto novel de epifitismo, dominado por parches densos de chusque (Chusquea sp.), arbustos de Miconia sp., Palicourea spp., Aphelandra sp., Piper spp., Besleria solanoides y herbáceas como Heliconia, Kohleria, Gasteranthus, Reldia grandiflora y Peperomia.

\section{Métodos de muestreo}

Trabajamos en 6 rutas diferentes de 2 a $3 \mathrm{~km}$, en tres parches de bosque montano que suman 20 ha. Obtuvimos los datos entre los meses de enero y julio del 2010, para los muestreos utilizamos 10 redes de niebla de $12 \mathrm{~m}$ x 2,5 m y $36 \mathrm{~mm}$ de ojo de malla, las cuales ubicamos individuales o en grupos de 2 a 5 mallas, distribuidas a lo largo de trochas de 200 a 300 m, en interior de bosque y borde de bosque.

Un grupo de dos observadores (JPT y DAG) operamos las redes durante cuatro días consecutivos, cambiando de lugar las mallas cada dos días para evitar la reducción de la tasa de captura $(7,10)$. Realizamos dos visitas mensuales cada 15 días durante 6 meses ubicando las mallas en diferentes sitios. Dejamos las redes abiertas entre las 06:00 y las 17:00 h según las condiciones climáticas, y las revisamos cada media o una hora de acuerdo con la actividad de las aves. Tomamos dimensiones del pico (longitud, ancho y alto), longitud del ala, longitud de la cola y longitud del tarso, además de la masa corporal de todos los individuos capturados (14). También registramos la presencia o ausencia de muda y la evidencia reproductiva (parche de incubación, protuberancia de la cloaca), acompañado por registro fotográfico. Luego marcamos cada ave con un pequeño corte en la rectriz externa y las liberamos en el mismo tipo de hábitat.

Un segundo grupo (AOD \& JSG) realizamos detecciones visuales y auditivas en puntos de conteo, durante los mismos días de trabajo con las redes de niebla. Montamos cuatro trayectos $1,5 \mathrm{~km}$ en coberturas de bosque y borde de bosque. En cada uno de estos ubicamos 10 puntos de conteo separados a una distancia de $150 \mathrm{~m}$. En cada punto de conteo, apuntamos todas las aves observadas y escuchadas con binoculares 10 x $50 \mathrm{~m}$ a una distancia radial menor a $30 \mathrm{~m}$, durante periodos de 10 min por punto. Cada trayecto se muestreo durante un día en la mañana (06:30-09:30 h) y en la tarde (15:00-16:00 h) cada 15 días durante seis meses. La clasificación taxonómica sigue a Gill \& Donsker (15).

Clasificamos las aves según su tamaño corporal según las categorías propuestas en Stiles \& Roselli (7) con base en una escala logarítmica de base 2 (I: $<5$ g, II: 6-12 g, III: 13-25 g, IV: 26-50 g, V: 51-100 g, VI: 101-200 g, VII: 201-400 g, VIII: $401-800$ g y IX $>801 \mathrm{~g}$ ); mientras que para el grupo trófico seguimos la clasificación de Renjifo (16): CA: Carroñeros, R/D: Rapaz diurna, FR: frugívoros, FR-I: Frugívorosinsectívoros, I: Insectívoro, NI: Nectarívoro, SE: Semillas. Por otra parte adoptamos la clasificación de gremios de forrajeo de Stiles \& Roselli (7): IAE: insectívoros aéreos; IHSM: insectívoros halconeadores de sotobosque y estrato medio; IHDB: insectívoros halconeadores del dosel y bordes de bosque; ISFS: insectívoros del suelo y el follaje de sotobosque; IFSM: insectívoros del follaje y ramas delgadas de estrato medio; IFDB; insectívoros de follaje del dosel y bordes; IIPA: insectos e invertebrados acuáticos o del borde de agua; ITR: insectos o vertebrados muy pequeños sobre o adentro de troncos y ramas gruesas; IGF: insectos grandes y vertebrados pequeños del follaje y ramas delgadas; CAV: cazadores (acecho o persecución) de vertebrados más grandes; FSSB: recogedores de frutos y semillas del suelo y sotobosque 
bajo; FPDB: consumidores de frutos pequeños del dosel y los bordes; FGDB: frutos más grandes del dosel y bordes; SPCG: semillas pequeñas de compuestas y gramíneas; NEC: consumidores de néctar.

\section{Análisis estadístico}

Evaluamos la efectividad del muestreo por medio curvas de acumulación de especies en donde las unidades de muestreo fueron las jornadas de trabajo (días), que incluyen la sumatoria del esfuerzo de 10 puntos de conteo en la mañana y 10 en la tarde y el trabajo con 10 redes de niebla durante todo el día. Para ello usamos los estimadores no paramétricos Chao 2, con el programa Estimates (17). Con la prueba de t de student comparamos el número de especies y número de individuos detectados en cada jornada de trabajo en los puntos de conteo y las capturas (12). Con la prueba de chi-cuadrado comparamos la proporción de especies capturadas y observadas en los diferentes grupos tróficos, tamaño corporal y gremio de forrajeo.

\section{RESULTADOS}

Encontramos 137 especies de aves de 34 familias con ambos métodos (Anexo 1), las familias mejor representadas fueron Tyrannidae (17), Trochilidae (16) Furnariidae (15) y Thraupidae (15). Nuestro esfuerzo de captura fue de 2630 horas-red, con el cual obtuvimos 608 capturas (54 recapturas), distribuidas en 81 especies de aves pertenecientes a 21 familias. Las familias más representadas fueron Tyrannidae (13), Furnariidae (12), Trochilidae (13) y Thraupidae (7). Las especies con mayor número de capturas fueron Adelomyia melanogenys (Fraser, 1840) con 91, Coeligena coeligena (Lesson, 1833) con 64, Myiothlypis coronata (Tschudi, 1844) con 46, Cinnycerthia olivascens Sharpe, 1882 con 38, y Phaethornis syrmatophorus Gould, 1852 con 36 (Anexo 1). Con un esfuerzo de 235 puntos de conteo equivalentes a 78,6 horas de observación obtuvimos 1436 registros pertenecientes a 106 especies de aves agrupadas en 30 familias. Las familias más representativas fueron: Tyrannidae (11), Furnariidae (9), Thraupidae (11) y Trochilidae (12). Las especies con mayor número de detecciones fueron: A. melanogenys con 119, Myiarchus cephalotes Taczanowski, 1880 con 92, Henicorhina leucophrys (Tschudi, 1844) con 89, Grallaria ruficapilla Lafresnaye, 1842 con 73, y Myadestes ralloides (d'Orbigny, 1840) con 66 (Anexo 1).

Con las redes de niebla obtuvimos registros de 31 especies que no detectamos en los puntos de conteo, mientras que en estos encontramos 55 especies que no logramos capturar. Con los puntos de conteo detectamos el doble de las especies capturadas en cada jornada de muestreo (observaciones: $22 \pm 6,8$; captura: $11,1 \pm 6,1 ; \mathrm{t}=6,05 ; \mathrm{p}<0,001 ; \mathrm{df}=50$ ), y tres veces un mayor número de detecciones que las capturas: (observaciones: 59,8 $\pm 31,4$; captura: $21,7 \pm 17,1 ; \mathrm{t}=$ $5,53 ; \mathrm{p}<0,0001 ; \mathrm{df}=50)$. La efectividad del muestreo con las capturas fue del $51,9 \%$, la curva de acumulación de especies mostró que los uniques y duplicates tendieron a estabilizarse pero no disminuyeron (Fig. 1A). De hecho, el número de especies estimada por Chao 2 presentó un incremento marcado conforme aumentó el número de jornadas de muestreo, además el alto intervalo de confianza de este estimador (IC $=109,4-278,8)$ indicó una estimación poco robusta (Figura. 1A). En contraste, los puntos de conteo presentaron una efectividad mayor, con una representatividad del 80,1\%, soportada por una disminución de los uniques y duplicates, una estabilización de las especies esperadas y un bajo intervalo de confianza (IC = 116,6-164,37; Fig. 1B). Al combinar ambos métodos, encontramos una representatividad del 78,2 \%, los uniques y duplicates permanecieron estables, el número de especies esperadas incrementó de forma gradual y no alcanzó una asíntota $(\mathrm{IC}=158,0-226,5$; Figura 1C).

Al comparar la distribución de la avifauna según su tamaño, encontramos que las aves pequeñas del grupo II estuvieron mejor representadas en las capturas que en los puntos de conteo, mientras que las especies de los grupos restantes, principalmente de las grandes (grupo VI al IX) se detectaron en los puntos de conteo (Fig. 2). Con respecto al grupo trófico, hubo una dominancia marcada en riqueza y abundancia de aves insectívoras en ambos métodos, seguido por las frugívoro-insectívoras (Fig. 3). Sin embargo las proporciones de riqueza y abundancia de los grupos tróficos fueron mayores en los puntos de conteo. En contraste, los nectarívoros estuvieron representados de forma equitativa entre las capturas y las observaciones, mientras que las rapaces diurnas y los consumidores de semillas e insectos estuvieron pobremente representados (Figura. 3).

La mayoría de las aves pertenecieron a más de un gremio de forrajeo (Anexo 1). Los puntos de conteo permitieron la detección de un mayor número de especies de los gremios del dosel (Fig. 4), como los frugívoros grandes (FGDB), los insectívoros de follaje (IFDB) 
y los insectívoros halconeadores (IHDB); especies poco conspicuas como los insectívoros del suelo y follaje del sotobosque (ISFS) y los consumidores de insectos y vertebrados pequeños de corteza de ramas $\mathrm{y}$ troncos (ITR) estuvieron mejor representadas en las observaciones (Fig. 4).

\section{DISCUSIÓN}

En nuestro estudio encontramos que las detecciones con los puntos de conteo fueron más eficientes en obtener un mayor número de especies durante cada jornada de trabajo con respecto a las redes de niebla, además de una acumulación de especies más alta y con menos esfuerzo, aspecto que ya ha sido ampliamente mencionado en la literatura $(1,4,7,9,12)$. Esto no solo causa sesgos en las estimaciones de riqueza, sino también en la identificación de patrones ecológicos de la avifauna (9). En nuestro caso, las redes fallaron en detectar la mayoría de aves del dosel y casi todas las especies de tamaño corporal grande que correspondieron principalmente a aves frugívoras grandes. Por lo tanto, no es posible hacer inferencias sobre la frecuencia de detección de estas especies (12). En contraste, las redes permitieron la detección de aves pequeñas del sotobosque, como los colibríes, o de especies relativamente grandes como los recogedores de frutos y semillas del sotobosque (Fig. 4, Anexo 1). Sin embargo las capturas también presentaron problemas en detectar especies insectívoras de estratos medios de los gremios de forrajeo ISFS y ITS, que estuvieron mejor representados en las observaciones. La mayoría de los miembros de estos gremios se unen a bandadas mixtas (18), y prefieren forrajear en estratos altos por lo que no podían ser capturadas. Además, algunas especies evadieron las mallas cuando las aves capturadas emitían llamados de alarma (p.e. Cinnycerthia olivascens).

La variación en la estructura del hábitat afecta la detección de algunas especies (1), las aves pueden ser capturadas con mayor frecuencia en las redes en las partes bajas del sotobosque que en las más altas o en el dosel del bosque a pesar de que la especie también es abundante en ambos hábitats (19). La distancia de vuelo y la frecuencia también pueden afectar la detección por redes de niebla (1). En nuestro estudio las especies más representativas y detectadas con las redes de niebla fueron Adelomyia melanogenys, Myiothlypis coronata, Cinnycerthia olivascens y Phaetornis syrmatophorus debido a que son especies confinadas a las partes bajas y medias del sotobosque según su historia natural (20). Además esta diferencia también puede deberse al esfuerzo de muestreo en este hábitat; esto debido en parte a que instalamos las redes en sitios como filos y pasos de montaña en tanto las aves del dosel como las del sotobosque son propensas a volar a poca altura y ser capturadas.

El uso de redes de niebla ha llevado a algunos investigadores (5), a identificar los factores que causan la variación en las tasas de captura. Estos incluyen el clima, la hora del día, lugar, la tensión de la red, la estructura del hábitat adyacente, y las especies furtivas, la territorialidad, patrones de vuelo y la migración (1, $5,21)$. Como se pudo evidenciar en nuestro estudio se presentan horas de mayor actividad desde las 7:00 $\mathrm{h}$ hasta las 10:00 h y entre las 12:00 h y las 14:00 h. Además también influyen el clima y la topografía que aunque no analizamos el efecto de estas variables en la tasa de capturas.

A pesar de ser más efectivo que las mallas, los puntos de conteo no proveen una descripción completa de la avifauna (12). Los puntos de conteo requieren de observadores familiarizados con las vocalizaciones de las aves y con la identificación en campo $(7,9,11$, 12). En nuestro caso el hecho de que 31 especies hayan pasado desapercibidas en los puntos de conteo es evidencia del efecto de nuestra falta de conocimiento de los cantos e identificación de las aves en condiciones de luz óptima y postura correcta. En contraste, en otros estudios se ha encontrado que casi todas las aves capturadas fueron registradas en observaciones o registros por puntos de conteo $(7,9,12)$.

La técnica de puntos de conteo requiere observadores expertos familiarizados con los cantos o las llamadas de cientos de especies de aves (22), aun así, podrían pasar desapercibidas especies que vocalizan poco o especies con hábitos discretos. También es frecuente que se pierda el registro de especies poco comunes o consideradas raras, además la baja densidad de algunas especies puede tener un efecto negativo en la vocalización de diversas o muchas especies que puede ser un importante componente de la avifauna (5, 23). Por lo tanto las redes de niebla se consideran más eficaces en la documentación de la presencia de estas especies. Por ejemplo en nuestro estudio, presentamos un nuevo reporte para los Andes centrales de Colombia de Grallaricula cucullata, una especie poco conspicua, y que aparentemente es mejor representada por capturas en redes (24), cuya vocalización es 
Revista de Investigaciones - Universidad del Quindío

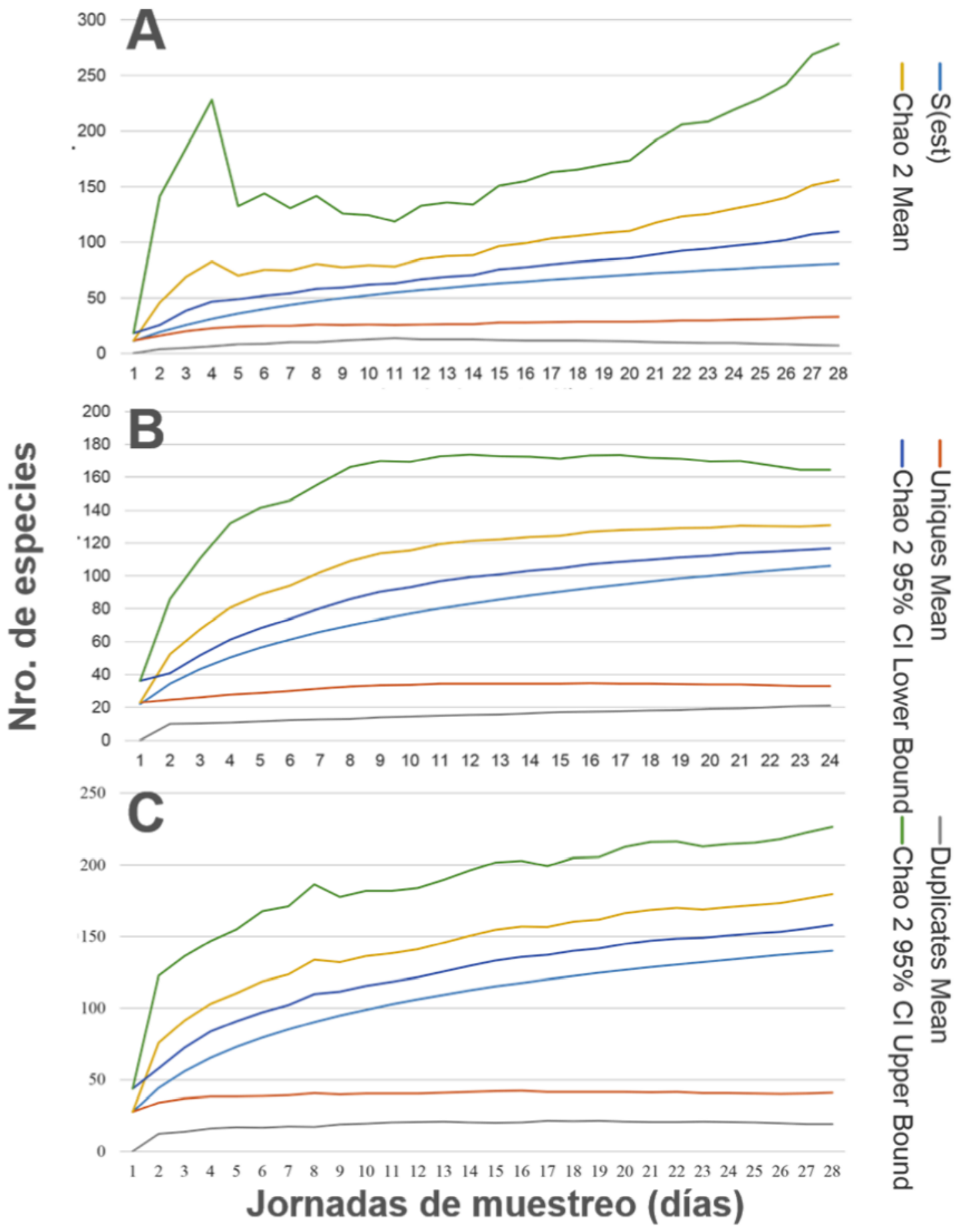

Figura 1. Curvas de acumulación de especies con el método de capturas (A), puntos de conteo (B) y combinación de ambos (C). 
Efectividad de las redes de niebla para determinar la riqueza de aves. Polanco et al.

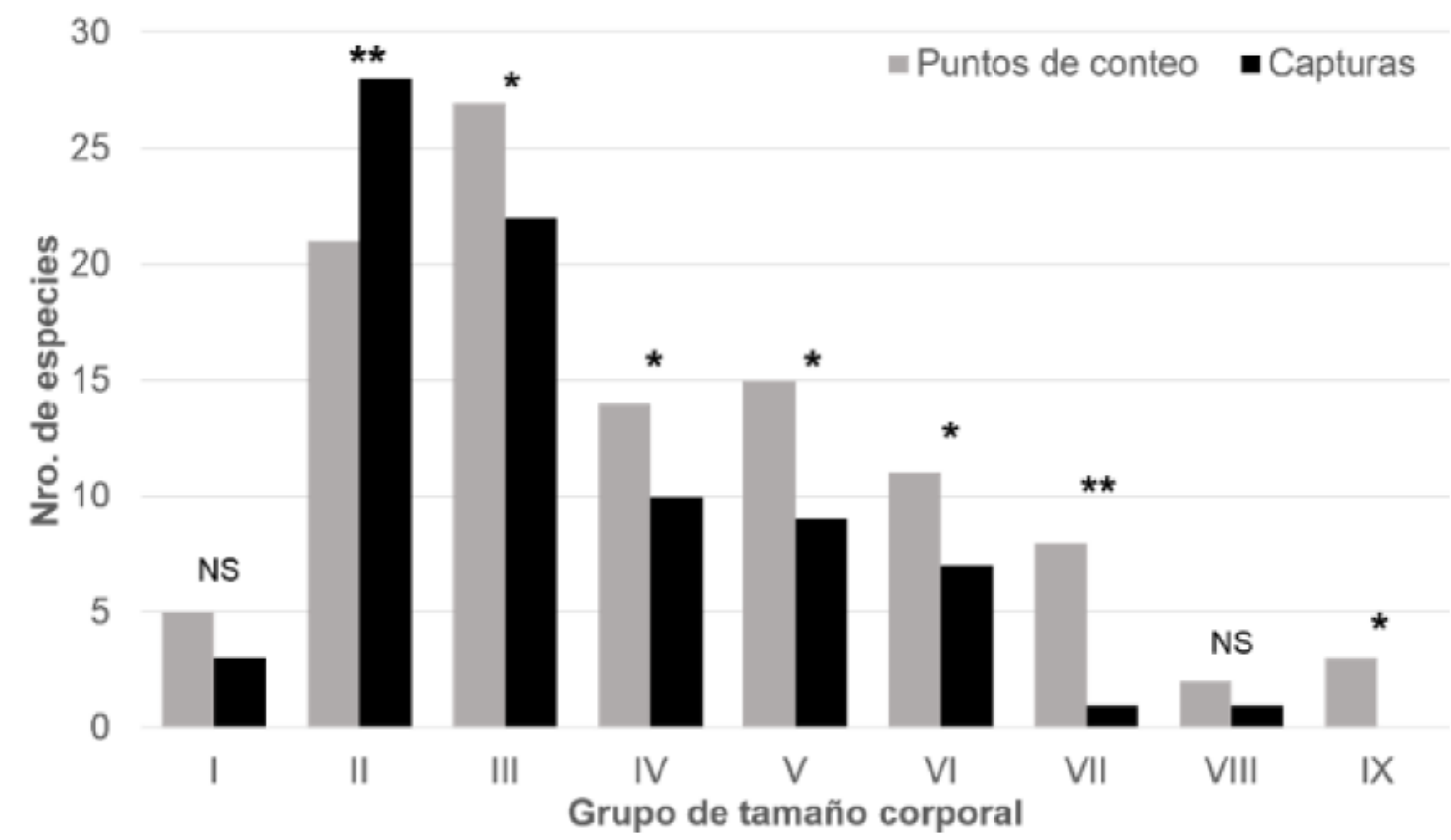

Figura 2. Distribución del número de especies según su tamaño corporal para cada tipo de método. Prueba de $X^{2}, *=\mathrm{p}<0,05, * *=\mathrm{p}<0,01, \mathrm{NS}=$ no significativo.

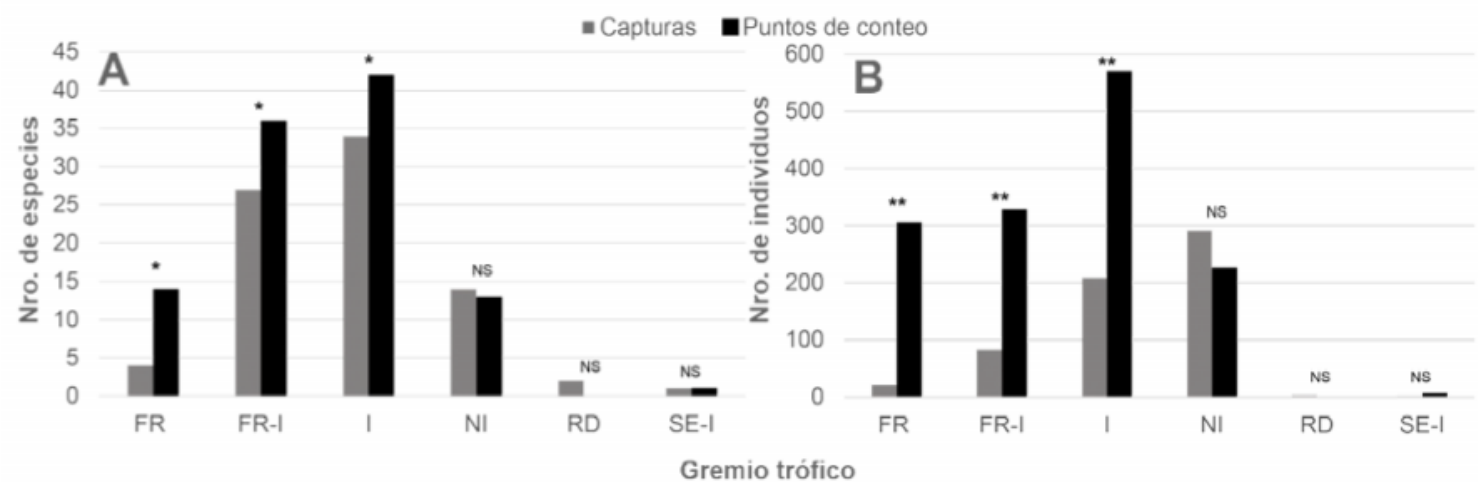

Figura 3. Distribución del número de especies y las abundancias de los grupos tróficos para cada tipo de método. Prueba de $X^{2}, *=\mathrm{p}<0,05, * *=\mathrm{p}<0,01, \mathrm{NS}=$ no significativo.

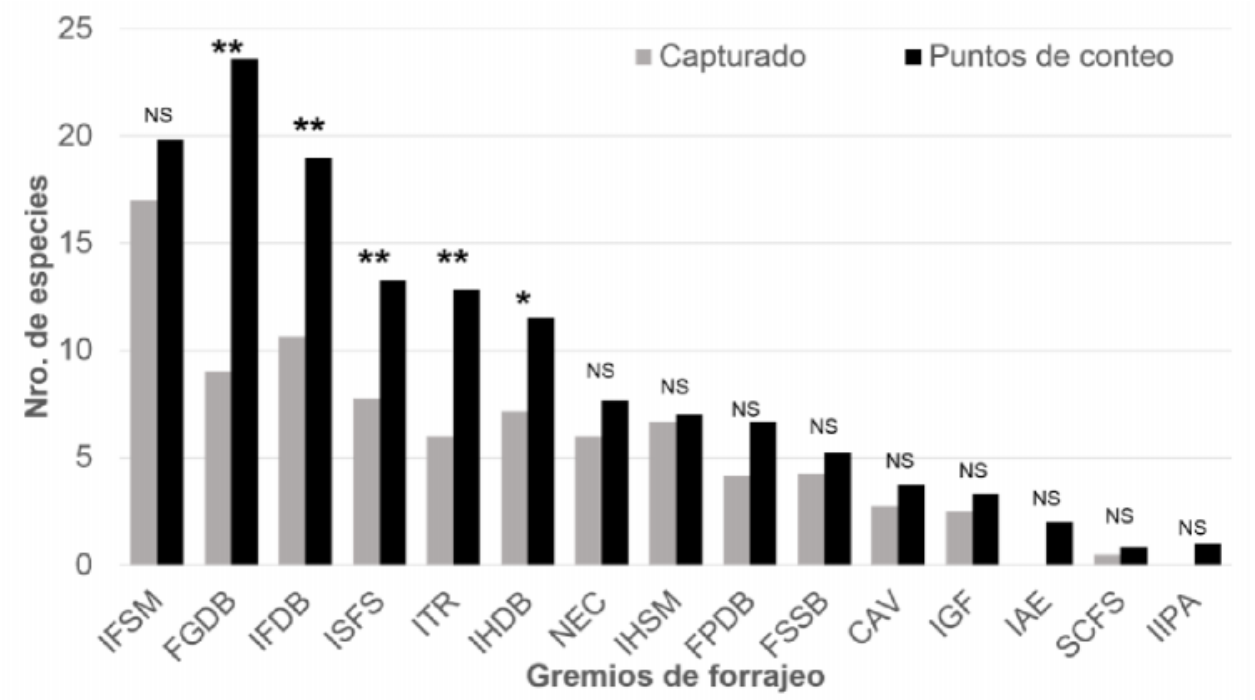

Figura 4. Distribución del número equivalente de especies según su gremio de forrajeo para cada tipo de método de muestreo. Prueba de $X^{2}, *=p<0,05, * *=p<0,01, \mathrm{NS}=$ no significativo. 
es desconocida, por lo que con el método de puntos de conteo no se detectó.

Los puntos de conteo de aves son un método estándar para el monitoreo de aves en todo el mundo, debido a su eficacia en diferentes tipos de hábitats (25). La inclusión de los puntos de conteo en evaluaciones rápidas de la diversidad, tienen la ventaja de concentrarse totalmente en las aves y sus hábitats al estar en un punto fijo y tener un mayor tiempo para identificar las especies y detectar aquellas que son cripticas y territoriales (por ejemplo, aves del sotobosque); además de permitir una asociación entre la presencia de las aves y su abundancia con las características estructurales y ambientales del hábitat (25). Sin embargo, una de sus limitaciones es que el muestreo de un solo punto puede dar una indicación poco precisa de la riqueza de especies de un área, principalmente de aquellas con alta riqueza, por lo que se requieren de muchas replicas para obtener una estimación relativamente confiable (26). Por lo anterior se ha sugerido la combinación de métodos como capturas con redes de niebla, detecciones por puntos de conteo y o trayectos, y grabaciones de vocalizaciones para detectar mayor número total de especies $(18,19,20,25)$.

En conclusión las observaciones por puntos de conteo es más efectivo que las redes de niebla, este ha sido documentado como uno de los más eficientes para obtener inventarios más completos en bosques tropicales $(7,12)$, aunque estas diferencias están determinadas por la experiencia de los observadores. Una ventaja de las redes de niebla sobre los puntos de conteo (observaciones visuales y auditivas) es que reduce el impacto de diferencias entre observadores en cuanto a la experiencia y capacidad de identificar aves en el campo. Las redes de niebla adquieren su mayor utilidad en la obtención de datos de morfometría, dieta, reproducción y muda, si estos fueran de interés para el inventario. En nuestro caso, el uso de las redes permitió registrar algunas especies que no se registraron con el método de puntos de conteo puesto que este último tienden a pasar por alto las especies furtivas que no vocalizan a menudo, también es frecuente que se pierda el registro de especies poco comunes o consideradas raras. Por lo anterior y al igual que otros autores $(1,9,11,18)$, recomendamos usar una combinación de estos métodos de muestreo, principalmente cuando los investigadores tengan poca experiencia en la identificación y reconocimiento de cantos. Ambos métodos pueden incrementar la probabilidad de detección, lo que le permite registrar un mayor número de especies y tener inventarios más completos que permitan conocer la diversidad de zonas con una biodiversidad tan elevada como la de los Andes Colombianos.

\section{AGRADECIMIENTOS}

Al apoyo de Smurfit Kappa cartón de Colombia y su personal que permitió la realización del presente trabajo. A nuestros compañeros Gloria Álvarez, Natalia Suarez, Eliana Quintana, Jenny Gómez, Yolanda y Luz Adriana por su colaboración en campo. A Mercedes Girón Vanderhuck, Enrique Arbeláez Cortes, Oscar Alexander Aguirre y Luis Hernando Hurtado Tobón por sus correcciones y sugerencias; a doña Luz y a Yiyo por su estadía y alimentación en la finca los andes vereda Boquia, Quindío. 
Efectividad de las redes de niebla para determinar la riqueza de aves. Polanco et al.

Anexo 1. Lista de la avifauna con el número de capturas, detecciones en los puntos de conteo y clasificación según el grupo trófico, gremio de forrajeo y peso corporal.

\begin{tabular}{|c|c|c|c|c|c|}
\hline Taxón & Capturas & $\begin{array}{l}\text { Puntos de } \\
\text { conteo }\end{array}$ & $\begin{array}{l}\text { Grupo } \\
\text { trófico }\end{array}$ & $\begin{array}{c}\text { Gremio de } \\
\text { forrajeo }\end{array}$ & $\begin{array}{c}\text { Grupo peso } \\
\text { corporal }\end{array}$ \\
\hline \multicolumn{6}{|l|}{ Tinamidae } \\
\hline Nothocercus julius (Bonaparte, 1854) & & 9 & FR-I & FSSB & VIII \\
\hline \multicolumn{6}{|l|}{ Cracidae } \\
\hline Chamaepetes goudotii (Lesson, 1828) & & 48 & FR & $\begin{array}{l}\text { FGDB, } \\
\text { FSSB }\end{array}$ & IX \\
\hline Penelope montagnii (Bonaparte, 1856) & & 2 & FR & $\begin{array}{l}\text { FGDB, } \\
\text { FSSB }\end{array}$ & IX \\
\hline Penelope perspicax Bangs, 1911 & & 3 & FR & $\begin{array}{l}\text { FGDB, } \\
\text { FSSB }\end{array}$ & IX \\
\hline \multicolumn{6}{|l|}{ Odontophoridae } \\
\hline Odontophorus hyperythrus Gould, 1858 & & 1 & FR-I & FSSB, ISFS & VI \\
\hline \multicolumn{6}{|l|}{ Accipitridae } \\
\hline Accipiter striatus Vieillot, 1808 & 2 & & $\mathrm{R} / \mathrm{D}$ & CAV & VI \\
\hline \multicolumn{6}{|l|}{ Columbidae } \\
\hline Patagioenas fasciata (Say, 1822) & & 6 & FR & $\begin{array}{l}\text { FPDB, } \\
\text { FGDB }\end{array}$ & VII \\
\hline \multicolumn{6}{|l|}{ Psittacidae } \\
\hline $\begin{array}{l}\text { Leptosittaca branickii von Berlepsch \& Stolzmann, } \\
1894\end{array}$ & & 8 & FR & FGDB & VII \\
\hline Psittacara wagleri (Gray, GR, 1845) & & 16 & FR & FGDB & VII \\
\hline Pionus seniloides (Massena \& Souancé, 1854) & 1 & 24 & FR & FGDB & VII \\
\hline Pionus chalcopterus (Fraser, 1841) & & 52 & FR & FGDB & VII \\
\hline Amazona mercenarius (Tschudi, 1844) & & 13 & FR & FGDB & VII \\
\hline \multicolumn{6}{|l|}{ Cuculidae } \\
\hline Piaya cayana (Linnaeus, 1766) & & 5 & FR-I & $\begin{array}{l}\text { IFDB, } \\
\text { FPDB }\end{array}$ & $\mathrm{V}$ \\
\hline \multicolumn{6}{|l|}{ Strigidae } \\
\hline Glaucidium jardinii (Bonaparte, 1855) & 1 & & $\mathrm{R} / \mathrm{D}$ & CAV & $\mathrm{V}$ \\
\hline \multicolumn{6}{|l|}{ Nyctibiidae } \\
\hline Nyctibius griseus (Gmelin, JF, 1789) & & 1 & I & IHDB & $\mathrm{V}$ \\
\hline \multicolumn{6}{|l|}{ Apodidae } \\
\hline Streptoprocne zonaris (Shaw, 1796) & & 7 & I & IAE & $\mathrm{V}$ \\
\hline \multicolumn{6}{|l|}{ Trochilidae } \\
\hline Phaethornis syrmatophorus Gould, 1852 & 36 & 2 & NI & NEC, IFSM & II \\
\hline Doryfera ludovicae (Bourcier \& Mulsant, 1847) & 1 & & NI & NEC, IHDB & II \\
\hline Colibri delphinae (Lesson, 1839) & & 1 & NI & NEC, IFSM & II \\
\hline Colibri thalassinus (Swainson, 1827) & 6 & 31 & NI & NEC, IHDB & II \\
\hline Colibri coruscans (Gould, 1846) & 3 & 30 & NI & NEC, IHDB & II \\
\hline Heliangelus exortis (Fraser, 1840) & 1 & & NI & NEC, IFSM & II \\
\hline Adelomyia melanogenys (Fraser, 1840) & 91 & 119 & NI & NEC, IFSM & I \\
\hline Aglaiocercus kingii (Lesson, 1832) & 7 & 3 & NI & $\begin{array}{l}\text { NEC, } \\
\text { IHSM, } \\
\text { IHDB }\end{array}$ & I \\
\hline Metallura tyrianthina (Loddiges, 1832) & & 12 & NI & $\begin{array}{l}\text { NEC, } \\
\text { IHSM, } \\
\text { IHDB }\end{array}$ & I \\
\hline
\end{tabular}


Revista de Investigaciones - Universidad del Quindío

Haplophaedia aureliae (Bourcier \& Mulsant, 1846)

Coeligena coeligena (Lesson, 1833)

Coeligena torquata (Boissonneau, 1840)

Lafresnaya lafresnayi (Boissonneau, 1840)

Ocreatus underwoodii (Lesson, 1832)

Heliodoxa rubinoides (Bourcier \& Mulsant, 1846)

Amazilia franciae (Bourcier \& Mulsant, 1846)

\section{Trogonidae}

Pharomachrus auriceps (Gould, 1842)

Trogon collaris Vieillot, 1817

Trogon personatus Gould, 1842

Momotidae

Momotus aequatorialis Gould, 1858

\section{Capitonidae}

Eubucco bourcierii (Lafresnaye, 1845)

\section{Ramphastidae}

Aulacorhynchus griseigularis Chapman, 1915

Aulacorhynchus haematopygus (Gould, 1835)

Andigena nigrirostris (Waterhouse, 1839)

\section{Picidae}

Melanerpes formicivorus (Swainson, 1827)

Picoides fumigatus (d'Orbigny, 1840)

Colaptes rubiginosus (Swainson, 1820)

Colaptes rivolii (Boissonneau, 1840)

Campephilus melanoleucos (Gmelin, JF, 1788)

Thamnophilidae

Drymophila striaticeps Chapman, 1912

\section{Grallariidae}

Grallaria alleni Chapman, 1912

Grallaria ruficapilla Lafresnaye, 1842

Grallaria nuchalis Sclater, PL, 1860

Grallaricula cucullata (Sclater, PL, 1856)

\section{Rhinocryptidae}

Scytalopus latrans Hellmayr, 1924

Scytalopus spillmanni Stresemann, 1937

\section{Furnariidae}

Dendrocincla tyrannina (Lafresnaye, 1851)

$\begin{array}{ccc}\text { FR } & \text { FGDB } & \text { VI } \\ \text { FR-I } & \text { FGDB, IGF } & \text { V } \\ \text { FR-I } & \text { FGDB, IGF } & \text { V }\end{array}$

FR-I IGT,ISFS, VI FGDB

FPDB, IV IFSM

$\begin{array}{ccc}\text { FR-I } & \text { FGDB, } & \text { VI } \\ & \text { CAV } & \\ \text { FR-I } & \text { FGDB, } & \text { VI } \\ & \text { CAV } & \\ \text { FR-I } & \text { FGDB, } & \text { VII } \\ & \text { CAV } & \end{array}$

1

$\begin{array}{ccr}\text { NI } & \text { NEC, IFSM } & \text { II } \\ \text { NI } & \text { NEC, } & \text { II } \\ & \text { IHSM, } & \\ & \text { IHDB } \\ \text { NI } & \text { NEC, } & \text { II } \\ & \text { IHSM, } & \\ & \text { IHDB } & \\ \text { NI } & \text { NEC, IFSM } & \text { II } \\ \text { NI } & \text { NEC, } & \text { I } \\ & \text { IHSM, } & \\ & \text { IHDB } & \\ \text { NI } & \text { NEC, } & \text { II } \\ & \text { IHSM, } & \\ & \text { IHDB } & \text { I } \\ \text { NI } & \text { NEC, IFSM }\end{array}$

I

$\mathrm{V}$

V

V

ITR V

ITR IV

ITR V

ITR $\mathrm{V}$

ITR VII

IFSM III

$\begin{array}{lc}\text { ISFS } & \mathrm{V} \\ \text { ISFS } & \mathrm{V} \\ \text { ISFS } & \mathrm{V} \\ \text { ISFS } & \text { III }\end{array}$

ISFS III

ISFS III

I

I

I

II


Efectividad de las redes de niebla para determinar la riqueza de aves. Polanco et al.

Glyphorynchus spirurus (Vieillot, 1819)

Dendrocolaptes picumnus Lichtenstein, MHK, 1820

Xiphocolaptes promeropirhynchus (Lesson, 1840)

Lepidocolaptes lacrymiger (Des Murs, 1849)

Campylorhamphus pusillus (Sclater, PL, 1860)

Premnornis guttuliger (Sclater, PL, 1864)

Premnoplex brunnescens (Sclater, PL, 1856)

Lochmias nematura (Lichtenstein, MHK, 1823)

Anabacerthia striaticollis Lafresnaye, 1840

Syndactyla subalaris (Sclater, PL, 1859)

Thripadectes holostictus (Sclater, PL \& Salvin, 1876)

Thripadectes virgaticeps Lawrence, 1874

Margarornis squamiger (d'Orbigny \& Lafresnaye, 1838)

Synallaxis azarae d'Orbigny, 1835

\section{Tyrannidae}

Phyllomyias cinereiceps (Sclater, PL, 1860)

Elaenia frantzii Lawrence, 1865

Zimmerius chrysops (Sclater, PL, 1859)

Pogonotriccus ophthalmicus Taczanowski, 1874

Mionectes olivaceus Lawrence, 1868

Mionectes striaticollis (d'Orbigny \& Lafresnaye, 1837)

Leptopogon rufipectus (Lafresnaye, 1846)

Hemitriccus granadensis (Hartlaub, 1843)

Poecilotriccus ruficeps (Kaup, 1852)

Myiophobus flavicans (Sclater, PL, 1861)

Empidonax virescens (Vieillot, 1818)

Pyrrhomyias cinnamomeus (d'Orbigny \& Lafresnaye, 1837)

Contopus sordidulus Sclater, PL, 1859

Silvicultrix diadema (Hartlaub, 1843)

Ochthoeca cinnamomeiventris (Lafresnaye, 1843)

Myiodynastes chrysocephalus (Tschudi, 1844)

Myiarchus cephalotes Taczanowski, 1880

\section{Cotingidae}

Pipreola riefferii (Boissonneau, 1840)

Pyroderus scutatus (Shaw, 1792)

Lipaugus fuscocinereus (Lafresnaye, 1843)

\section{Tityridae}

Pachyramphus versicolor (Hartlaub, 1843)

Vireonidae

\section{6}

5

(n)

1

2

7

1

1

1

2

2

35

1

3

(1)

3

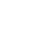

1

(1)

92

11

20

7
ITR

ITR

ITR

ITR

ITR

IFSM

ISFS

IIPA

IFSM

IFSM, IFDB

IFSM, IGF, ISFS

IFSM, IGF, $\quad$ V

ISFS

ITR, IFDB III

ISFS, IFSM III

IFDB II

FPDB, II

IFSM

FPDB, II

FR-I FPDB, II

$\begin{array}{lll}\text { I } & \text { IHDB } & \text { II }\end{array}$

FR-I FPDB, II

FR-I FPDB, II

IFDB

IHDB II

IFSM II

IHSM II

IHSM II

IFSM II

IHDB II

IHDB II

IHSM II

IHSM III

IHDB, IV

FPDB

IHSM III

FGDP, IV

FPDB

FR FGDB VIII

FR FGDB VI

FR-I IFDB, III

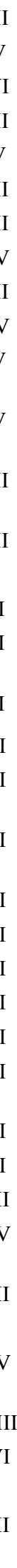


Revista de Investigaciones - Universidad del Quindío

Cyclarhis nigrirostris Lafresnaye, 1842

Vireo leucophrys (Lafresnaye, 1844)

1

\section{Corvidae}

Cyanolyca armillata (Gray, GR, 1845)

Cyanocorax yncas (Boddaert, 1783)

\section{Hirundinidae}

Notiochelidon cyanoleuca (Vieillot, 1817)

Troglodytidae

Troglodytes solstitialis Sclater, PL, 1859

Henicorhina leucophrys (Tschudi, 1844)

Cinnycerthia olivascens Sharpe, 1882

\section{Turdidae}

Myadestes ralloides (d'Orbigny, 1840)

Catharus fuscater (Lafresnaye, 1845)

Catharus ustulatus (Nuttall, 1840)

Turdus leucops Taczanowski, 1877

Turdus fuscater d'Orbigny \& Lafresnaye, 1837

Turdus serranus Tschudi, 1844

\section{Thraupidae}

Hemispingus atropileus (Lafresnaye, 1842)

Hemispingus frontalis (Tschudi, 1844)

Hemispingus melanotis (Sclater, PL, 1855)

Chlorornis riefferii (Boissonneau, 1840)

Anisognathus somptuosus (Lesson, 1831)

Thraupis cyanocephala (d'Orbigny \& Lafresnaye, 1837)

Tangara heinei (Cabanis, 1850)

Tangara vassorii (Boissonneau, 1840)

Tangara xanthocephala (Tschudi, 1844)

Tangara nigroviridis (Lafresnaye, 1843)

Tangara labradorides (Boissonneau, 1840)

Tangara arthus Lesson, 1832

Diglossa albilatera Lafresnaye, 1843

Diglossa cyanea (Lafresnaye, 1840)

Catamblyrhynchus diadema Lafresnaye, 1842

\section{Emberizidae}

Zonotrichia capensis (Statius Müller, PL, 1776)

Arremon brunneinucha (Lafresnaye, 1839)

Arremon assimilis (Boissonneau, 1840)

Atlapetes albinucha (Lafresnaye \& d'Orbigny, 1838)
IAE

III

17

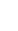

FR-I IFDB, IV

FR-I IFDB, FPDB IV

FR-I FPDB, IFDB III

FR-I FPDB, IFDB III

FR-I FPDB, IFDB III

FR-I FPDB, IFDB III

FR-I FPDB, IFDB III

FR-I FPDB, IFDB III

NI NEC, IFDB, II
FPDB

NI NEC, IFDB, III

$$
\text { FPDB }
$$$$
\text { FR-I IFSM, FPDB III }
$$

$\begin{array}{lll}\text { SE-I } & \text { SCFS, IFDB } & \text { III } \\ \text { FR-I } & \text { ISFS, FSSB } & \text { IV }\end{array}$

16

FR-I ISFS, FSSB IV

ISFS, FSSB
FR-I IFSM, IFDB, FPDB 
Efectividad de las redes de niebla para determinar la riqueza de aves. Polanco et al.

Atlapetes schistaceus (Boissonneau, 1840)

Chlorospingus flavopectus (Lafresnaye, 1840)

Chlorospingus canigularis (Lafresnaye, 1848)

Cardinalidae

Saltator cinctus Zimmer, JT, 1943

Parulidae

Mniotilta varia (Linnaeus, 1766)

Myiothlypis coronata (Tschudi, 1844)

Setophaga pitiayumi (Vieillot, 1817)

Basileuterus tristriatus (Tschudi, 1844)

Setophaga fusca (Statius Müller, PL, 1776)

Cardellina canadensis (Linnaeus, 1766)

Myioborus miniatus (Swainson, 1827)

Myioborus ornatus (Boissonneau, 1840)

\section{Icteridae}

Cacicus leucoramphus (Bonaparte, 1845)

Amblycercus holosericeus (Deppe, 1830)

\section{Fringillidae}

Euphonia xanthogaster Sundevall, 1834

Chlorophonia cyanea (Thunberg, 1822)

\begin{tabular}{|c|c|c|c|c|}
\hline & 11 & FR-I & $\begin{array}{l}\text { IFSM, IFDB, } \\
\text { FPDB }\end{array}$ & IV \\
\hline 1 & 27 & FR-I & IFDB, FPDB & III \\
\hline 1 & 3 & FR-I & IFDB, FPDB & III \\
\hline \multirow[t]{2}{*}{3} & & FR-I & IFDB, FPDB & III \\
\hline & 1 & I & IFDB, IFSM & II \\
\hline \multirow[t]{2}{*}{46} & 27 & I & ISFS, IFSM & III \\
\hline & 4 & I & IFDB, IFSM & II \\
\hline 1 & 2 & I & ISFS, IFSM & III \\
\hline 1 & 5 & I & IFDB, IFSM & II \\
\hline 7 & & I & IFDB, IFSM & II \\
\hline 9 & 55 & I & IHDB & II \\
\hline 2 & 2 & I & IHDB & II \\
\hline 1 & 3 & FR-I & $\begin{array}{l}\text { IGF, ITR, } \\
\text { IFDB }\end{array}$ & $\mathrm{V}$ \\
\hline \multirow[t]{3}{*}{4} & & FR-I & $\begin{array}{l}\text { IGF, ITR, } \\
\text { IFDB }\end{array}$ & $\mathrm{V}$ \\
\hline & 1 & I & FPDB & III \\
\hline & 1 & FR-I & $\begin{array}{l}\text { IFDB, } \\
\text { FPDB }\end{array}$ & III \\
\hline
\end{tabular}

\section{BIBLIOGRAFÍA}

1. Remsen J V, D A Good. Misuse of data from mist-net captures to assess relative abundance in bird populations. Auk 1996; 113(2):381-398.

2. Lövei G L, T Csörgõ, G Miklay. Capture efficiency of small birds by mist nets. Ornis Hungarica 2001; 11: 19-25. 2001

3. Pardieck K, R B Waide. Mesh size as a factor in avian community studies using mist nets. Journal of Field Ornithology 1992; 63: 250-255.

4. Karr J R. On the use of mist nets in the study of bird communities. Inland Bird Banding 1979; 51:1-10.

5. Karr J R. Surveying birds with mist nets. Studies in Avian Biology 1981; 6:62-67.

6. Jenii L, M Leuenberger, F Rampazzi.Capture efficiency of mist nets with comments on their role in the assessment of passerine habitat use. Field Ornithology 1996; 67(2): 263-274.

7. Stiles F G, L. Roselli. Inventario de las aves de un bosque altoandino: comparación de dos métodos. Caldasia 1998; 20(1): 29-43.

8. Mayerhofer M S, M R Hudson, M A Gahbauer. Net avoidance in passerine birds, 2008. Consultado en: http://www.migrationresearch.org/mbo/documents/NetavoidanceMay2008.pdf

9. Stiles F G, C I Bohórquez. Evaluación de la avifauna de las Quinchas. Caldasia 2000; 22 (1):61-69.

10. Villareal H, M Álvarez, S Córdoba, F Escobar, G Fagua, F Gast, H Mendoza, M Ospina, A M Umaña. Manual de métodos para el desarrollo de inventarios de biodiversidad. Programa de inventarios de biodiversidad. Instituto Alexander von Humboldt. Bogotá; 2004

11. Rappole J H, W J Mcsheaa, H V Rivera. Estimation of species and number sinupland avian breeding 
communities. Journal of Field Ornithology 1993; 64:55-70.

12. Whitman A, J Hagan, N V Brokaw. A comparison of two bird survey techniques used in a subtropical forest. The Condor 1997; 99:955-965

13. Arroyabe M A, Gómez L, Marín L A Restrepo M, Ortiz C, Gómez H, Cardona C y Muñoz N. Conservación de la avifauna en el AICA la patasola a través de una estrategia de zonificación y formulación de un plan de manejo. Instituto de Investigación de Recursos Biológicos Alexander von Humboldt. Bogotá; 2005.

14. Baldwin S H, Oberholser Y, Worley L. Measurements of birds. Scientific Publications Cleveland Museum Natural History 1931; 2:1-165.

15. Gill F, D Donsker (Eds). IOC World Bird List (v 4.1). 2014.doi 10.14344/IOC.ML.5.1

16. Renjifo L M. Composition changes in a subandean avifauna after long-term forest fragmentation. Conservation Biology 1999; 13 (5): 1124-1139.

17. Colwell R K. Estimates: statistical estimation of species richness and shared species from samples; 2004

18. Arbeláez-Cortés E, H A Rodríguez-Correa, M. Restrepo-Chica. Mixed bird flocks: patterns of activity and species composition in a region of the Central Andes of Colombia. Revista Mexicana de Biodiversidad 2011; 82: 639-651.

19. Petit D R, L J Petit, K G Smith. Habitat associations of migratory birds over wintering in Belize, Central America. In J M Hagan III, D W Johnston [eds.], Ecology and conservation of Neotropical migratory land birds. Smithson. Inst. Press. Pp 247-256. Washington, DC; 1992.

20. Hilty S L, W L Brown. Guía de las aves de Colombia. Princeton: Princeton University Press; 1986.

21. Keyes B E, Y C E Grue. Capturing birds with mist nets: a review. North American Bird Bander 1982; 7: 2-14.

22. Beehler B M, J B Sengo, C Filardi, K Merg. Documenting the lowland rainforest avifauna in Papua New Guinea--effects of patchy distributions, survey effort and methodology. Emu 1995; 95:149-161.

23. Rappole J H, G L Waggerman. Calling males as an index of density for breeding white-winged doves. Wildlife Society Bulletin 1986; 14:151-155

24. Marín-Gómez O H, J Polanco, D Giraldo, A Ospina. A new population of the Hooded Antpitta (Grallaricula cucullata: Grallaridae) for the Colombian Central Andes. Acta Biológica Colombiana 2015; 20(1): 229-232

25. Bibby C J, D A Hill, N D Burgess, S Mustoe. Bird census techniques. 2nd. Edition. Academic Press. London; 2000

26. O' Dea N J, M Watson, R J Whittaker. Rapid assessment in conservation research: a critique of avifaunal assessment techniques illustrated by Ecuadorian and Madagascan case study data. Diversity and Distributions 2004; 10 (1): 55-63. 